\section{Unclear Value of Positional MR Angiography in Evaluating Cerebral Venous Outflow Hemodynamics}

We read with interest the article entitled "Positional Venous MR Angiography: An Operator-Independent Tool to Evaluate Cerebral Venous Outflow Hemodynamics," originally published in the American Journal of Neuroradiology on-line on November 3, 2011 . $^{1}$

We believe that the authors did not consult relevant references previously reporting some of the key issues investigated by their study; the authors state that strictures of the jugular veins are common in healthy subjects at the level of the craniocervical junction in the supine position, which has already been noticed in previous work. $^{2-4}$ In these studies, a high incidence of venous stenoses at the level of the lateral mass of the atlas (C1) and at the level of the inferior thyroid gland (C6/C7), in a cohort of healthy subjects and patients with multiple sclerosis (MS), was a physiologic finding (mostly due to compression of the adjacent structures) on the basis of the test-retest reproducibility results. ${ }^{4}$ It was reported by Niggemann et $\mathrm{al}^{1}$ that criterion 3 on Doppler sonography ${ }^{5}$ represents a "proximal (superior) stricture of the IJV [internal jugular vein]," whereas criterion 3 referred to the "high resolution B-mode evidence of proximal IJV stenoses." The term "proximal" refers to relationship to the heart, indicating that stenosis is considered at the level of the thyroid or lower IJV area, not the upper craniocervical junction area.

Furthermore, the authors compared the IJV strictures in the supine-versus-upright position, which is not possible because in the supine position, the main venous extracranial outflow is through IJVs, so the signal-intensity flow in the IJVs in the upright position is very low or absent. This makes reliable determination of vein morphology changes difficult. ${ }^{5}$ On the basis of their results, which probably represent physiologic findings, ${ }^{2-4}$ they make a conclusion regarding the validity of the use of MR venography stenosis findings as a criterion for detection of chronic cerebrospinal venous insufficiency. In our opinion, this is an erroneous and misleading statement because previous reproducibility studies have shown that stenoses at the reported levels of $\mathrm{C} 1 / \mathrm{C} 6 / \mathrm{C} 7$ present with high variability in both patients with MS and healthy controls. ${ }^{4}$

Although the authors mentioned briefly that veins are prone to collapse, they do not discuss this in light of their findings, especially with respect to change toward the upright position, as previously described. ${ }^{2-4}$ In addition, by using only the $2 \mathrm{D}$ time-of-flight technique, they were not able to alleviate some of the usual MR venography artifacts and provide more detailed flow information. It was previously reported that assessment of reflux into the venous system by MR venography is not reliable even at $3 \mathrm{~T}^{2-4}$ In addition, criterion 1 on Doppler sonography ${ }^{5}$ represents reflux in the supine and upright position, not the supine or upright position, as reported by the authors. Also, the low strength of their MR imaging machine, $0.6 \mathrm{~T}$, and thicker sections used, $3.5 \mathrm{~mm}$, which led to poor contrast and spatial resolution in comparison with standard MR imaging devices with 1.5-3T strength and section thicknesses of $1.5 \mathrm{~mm}$, could have influenced their results considerably.

\section{References}

1. Niggemann P, Seifert M, Förg A, et al. Positional Venous MR Angiography: An Operator-Independent Tool to Evaluate Cerebral Venous Outflow Hemodynamics. AJNR Am J Neuroradiol 2011;33:246-51

2. Hojnacki D, Zamboni P, Lopez-Soriano A, et al. Use of neck magnetic resonance venography, Doppler sonography and selective venography for diagnosis of chronic cerebrospinal venous insufficiency: a pilot study in multiple sclerosis patients and healthy controls. Int Angiol 2010;29:127-39

3. Zivadinov R, Galeotti R, Hojnacki D, et al. Value of MR venography for detection of internal jugular vein anomalies in multiple sclerosis: a pilot longitudinal study. AJNR Am J Neuroradiol 2011;77:138-44

4. Zivadinov R, Lopez-Soriano A, Weinstock-Guttman B, et al. Use of MR venography for characterization of the extracranial venous system in patients with multiple sclerosis and healthy control subjects. Radiology 2011;258:562-70

5. Zamboni P, Galeotti R, Menegatti E, et al. Chronic cerebrospinal venous insufficiency in patients with multiple sclerosis. J Neurol Neurosurg Psychiatry 2009;80:392-99

K. Dolic

K. Marr

Buffalo Neuroimaging Analysis Center State University of New York

R. Zivadinov

Buffalo Neuroimaging Analysis Center State University of New York

The Jacobs Neurological Institute, Department of Neurology University at Buffalo, State University of New York Buffalo, New York

http://dx.doi.org/10.3174/ajnr.A2991 